Suporte social e contato intergeracional: estudando idosos com alterações cognitivas

\title{
Social support and intergenerational contact: studying elderly patients with cognitive alterations
}

Bruna Moretti Luchesi ${ }^{1}$, Tábatta Renata Pereira de Brito ${ }^{2}$, Reijane Salazar Costa ${ }^{3}$, Sofia Cristina lost Pavarini ${ }^{4}$

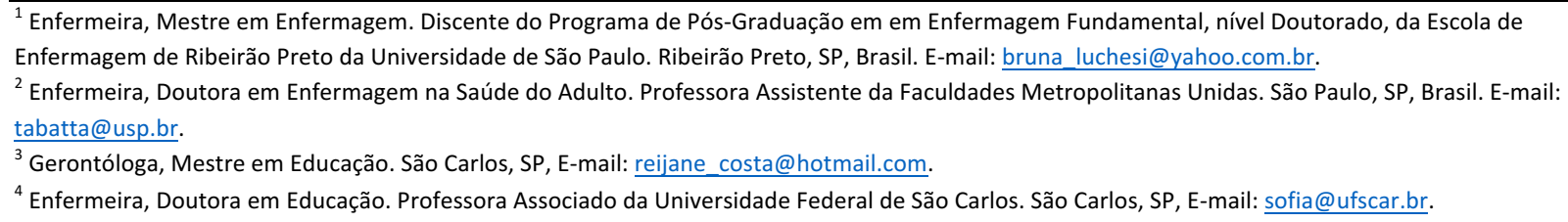

\section{RESUMO}

Este estudo teve por objetivo verificar a relação entre apoio social de idosos com alterações cognitivas que residem em diferentes contextos de vulnerabilidade social e a presença de crianças vivendo no mesmo domicílio. Trata-se de um estudo descritivo, transversal, que avaliou 85 idosos residentes em diferentes contextos de vulnerabilidade social, e que apresentaram resultado no Mini Exame do Estado Mental abaixo da nota de corte. Todos os cuidados éticos foram observados. Foram aplicados instrumento de caracterização sociodemográfica e familiar, Mini Exame do Estado Mental e o Medical Outcomes Study (MOS). Os resultados revelaram bons níveis de apoio social recebidos pelos idosos e correlação estatisticamente significativa entre a dimensão de apoio emocional e a presença de crianças no domicílio $(t=2,16$, sendo $p<0,01)$. Esses resultados possibilitam conhecer melhor a relação entre idosos e crianças ao revelar que o contato intergeracional favorece a percepção de apoio social satisfatório pelos idosos.

Descritores: Idoso; Criança; Apoio Social; Família; Enfermagem Geriátrica.

\section{ABSTRACT}

The aim of this study was to investigate the relationship between social support to elderly patients with cognitive alterations in different contexts of social vulnerability and the presence of children living in the same household. A descriptive, cross-sectional study assessed 85 elderly individuals in different contexts of social vulnerability; all of them presented results below the cut-off point in the mini mental state examination. All ethical guidelines were respected. A sociodemographic and family characterization tool, the mini mental state examination, and the Medical Outcomes Study (MOS) were applied. The results showed good levels of social support received by elderly individuals and a statistically significant correlation between the extent of emotional support and the presence of children in the household $(t=2.16, p<0.01)$. These results enable a better understanding on the relationships between elderly people and children by evidencing that intergenerational contact favors the perception of satisfactory social support by the elderly.

Descriptors: Aged; Child; Social Support; Family; Geriatric Nursing. 


\section{INTRODUÇÃO}

Em todo o mundo, o número de pessoas com 60 anos ou mais está crescendo mais rapidamente do que o de qualquer outra faixa etária. Especificamente nos últimos 60 anos, o número de idosos na população brasileira tem aumentado significativamente, sendo que em 1950, representavam 4,9\% da população total e em 2010, já eram 19,6 milhões, correspondente a $10,2 \%$ da população. Considerando que nos próximos 40 anos esse grupo crescerá a uma taxa de 3,2\% ao ano, o número de idosos passará a ser 64 milhões em 2050, o que corresponderá a $29,7 \%$ do total. Porcentagem essa, muito próxima a do Japão, atualmente o país com maior parcela de idosos do mundo(1).

Com o envelhecimento populacional, a interação entre as pessoas e o convívio intergeracional aumentam, resultando em uma sociedade composta por quatro gerações, já que muitos idosos têm a oportunidade de conhecer seus netos e bisnetos ${ }^{(2)}$. Além disso, tem sido cada vez mais comum a existência de arranjos familiares onde os idosos residem com gerações mais jovens.

Segundo dados do Estudo SABE (Saúde, Bem- Estar e Envelhecimento), realizado em São Paulo em 2006, 30,3\% dos idosos residem com filhos, $23,9 \%$ somente com cônjuge, $20,4 \%$ com filhos e netos, $13,3 \%$ sozinhos e $12,1 \%$ em outros arranjos domiciliares ${ }^{(3)}$. Em Ribeirão Preto-SP, dos 147 idosos com 80 anos e mais entrevistados, 10,9\% moravam em arranjos trigeracionais e $2 \%$ residiam com os netos ${ }^{(4)}$.

Considerando que com o envelhecimento a demanda por cuidados do idoso pode aumentar, e que a família é a principal fonte de ajuda, a presença de crianças morando com idosos pode influenciar as trocas de apoio no domicílio $^{(5)}$. Tais trocas interpessoais, envolvendo afeto, afirmação e ajuda, definem o termo "apoio social" ${ }^{(6)}$. As redes sociais, por sua vez, constituem o conjunto de relacionamentos que cada indivíduo mantém, sendo o

\footnotetext{
${ }^{1}$ Índice divulgado pela Fundação Sistema Estadual e Análise de Dados no
ano de 2007.

${ }^{1}$ Índice divulgado pela Fundação Sistema Estadual e Análise de Dados no
ano de 2007. Rev. Eletr. Enf. [Internet]. 2015 jul./set.;17(3). Disponível em: http://dx.doi.org/10.5216/ree.v17i3.25597. redes $^{(7)}$. idosos.

\section{METODOLOGIA}

De maneira geral, pessoas que se envolvem em redes sociais vivem mais e com melhor saúde do que as pessoas que não se envolvem em redes ${ }^{(8)}$. O efeito das redes sociais sobre a saúde dos idosos tem sido demonstrado, principalmente na qualidade de vida, bem-estar subjetivo, funcionalidade, mortalidade e declínio $\operatorname{cognitivo}^{(8-9)}$. Quanto aos aspectos cognitivos, o apoio social funciona como um fator de proteção contra o declínio cognitivo. O envolvimento em redes sociais ajuda a manter o idoso autônomo dentro do seu contexto familiar e sociocultural, o que é fundamental para as funções cognitivas e para seu bem-estar psicológico ${ }^{(10)}$.

Há uma complexidade emocional permeando o relacionamento entre idosos, filhos adultos e netos, e a qualidade desse relacionamento está associada às condições de saúde física e mental dos idosos ${ }^{(11)}$. Nesse sentido, o relacionamento entre idosos e crianças aumenta as possibilidades de troca de apoio social na velhice, o que pode melhorar a qualidade de vida dos

Considerando-se, portanto, que o número de crianças morando com idosos é bastante frequente e que o apoio social pode ser um fator protetor para o declínio cognitivo, o objetivo desse estudo foi verificar a relação entre apoio social de idosos com alterações cognitivas e a presença de crianças vivendo no mesmo domicilio.

Estudo descritivo, transversal e quantitativo, realizado em um município localizado na região central do Estado de São Paulo.

Foram sujeitos dessa pesquisa idosos (pessoas com mais de 60 anos) ( $n=85)$, cadastrados em Unidade de Saúde da Família (USF) localizadas em diferentes regiões de vulnerabilidade social de acordo com o Índice Paulista de Vulnerabilidade Social (IPVS) ${ }^{1}$. 
Os critérios de inclusão foram: ter mais de 60 anos, ser cadastrado em uma das USF da cidade que possuem classificação no IPVS, apresentar resultado no MiniExame do Estado Mental (MEEM) abaixo da nota de corte, não possuir comprometimento grave de linguagem ou compreensão e aceitar participar do estudo.

Para seleção dos sujeitos foi utilizado um banco de dados constituído de avaliações de 755 idosos das USF com classificação no IPVS da cidade. Destes, 195 apresentaram resultado abaixo da nota de corte no MEEM, constituindo assim os idosos com indicativo de alterações cognitivas. Descartando as perdas por óbitos e migração de domicílio, o presente estudo reavaliou 101 idosos. Após reaplicação do MEEM foram excluídos 16 idosos que apresentaram resultado acima da nota de corte, o que totalizou uma amostra final de 85 idosos.

Foram realizadas avaliações individuais e domiciliares, a partir de agendamento prévio com os sujeitos, entre os meses de agosto e novembro de 2009. A coleta de dados compreendeu:

- Caracterização sociodemográfica e familiar dos idosos;

- Aplicação do Medical Outcomes Study (MOS), instrumento utilizado para avaliação do apoio social oferecido aos idosos. Elaborado originalmente para o MOS, um estudo com 2.987 adultos, usuários de serviços de saúde em Boston, Chicago e Los Angeles; e traduzido para o português e validado em estudo realizado com a população do Rio de Janeiro - Estudo PróSaúde ${ }^{(12)}$. O questionário é composto por 19 itens, abrangendo cinco dimensões funcionais de apoio social: material, afetivo, emocional, interação social positiva e de informação. 0 apoio social é medido por meio da frequência com que o idoso dispõe de cada uma das dimensões. De acordo com as respostas, chegase a um escore final para cada uma das dimensões, que varia de 20 a 100 pontos, e quanto maior o escore alcançado, maior o nível de apoio social.

- Reaplicação do MEEM, instrumento usado para rastreamento de déficit cognitivo ${ }^{(13)}$ que serviu como critério de inclusão do idoso no estudo. As notas de corte foram adaptadas de acordo com o grau de escolaridade do idoso. Utilizou-se: 18 pontos para analfabetos, 21 pontos para um a três anos de escolaridade, 24 pontos para quatro a sete anos de escolaridade e 26 pontos para oito anos ou mais ${ }^{(14)}$.

Os dados foram analisados por meio de estatística descritiva e correlacional. Aplicou-se o teste de normalidade de Shapiro-Wilk e o Teste-t, com nível de significância de $5 \%$ ( $p$-value $<0,05)$.

Foram respeitadas as recomendações da Resolução 466/2012 regulamentada pelo Conselho Nacional de Saúde, que trata de pesquisas envolvendo seres humanos. O projeto de pesquisa foi aprovado pelo Comitê de Ética em Pesquisa da Universidade (Processo 119/2009), a coleta de dados foi autorizada pela Secretaria Municipal de Saúde do município e só teve início após leitura e assinatura do Termo de Consentimento Livre e Esclarecido.

\section{RESULTADOS}

A maioria dos idosos era do sexo feminino, com idade entre 70-79 anos, analfabeto e viúvo. Em relação ao local de moradia, a maioria residia em região de média, baixa ou muito baixa vulnerabilidade social. A renda individual do idoso foi na grande maioria dos casos, menor do que um salário mínimo.

As características sociodemográficas dos sujeitos podem ser observadas na Tabela 1. 
Tabela 1: Caracterização sociodemográfica dos idosos entrevistados. São Carlos, SP, Brasil, 2010.

\begin{tabular}{|c|c|c|}
\hline & Característica & $\%$ \\
\hline \multirow{2}{*}{ Sexo } & Feminino & 75,3 \\
\hline & Masculino & 24,7 \\
\hline \multirow{4}{*}{ Idade } & $60-69$ anos & 23,5 \\
\hline & $70-79$ anos & 43,5 \\
\hline & $80-89$ anos & 23,5 \\
\hline & 90 anos ou mais & 9,5 \\
\hline \multirow{7}{*}{ Escolaridade } & Analfabeto & 38,8 \\
\hline & 1 a 3 anos de escolaridade & 28,2 \\
\hline & 4 a 7 anos de escolaridade & 27,1 \\
\hline & 8 anos de escolaridade & 3,5 \\
\hline & 9 a 11 anos de escolaridade & 1,2 \\
\hline & 12 anos de escolaridade & 0 \\
\hline & mais de 12 anos de escolaridade & 1,2 \\
\hline \multirow{4}{*}{ Estado civil } & Viúvo & 51,8 \\
\hline & Casado/ Amasiado & 35,3 \\
\hline & Solteiro & 7,1 \\
\hline & Separado/ Divorciado & 5,8 \\
\hline \multirow{2}{*}{ IPVS } & Alta e muito alta vulnerabilidade social & 45,9 \\
\hline & Média, baixa, muito baixa vulnerabilidade social & 54,1 \\
\hline \multirow{2}{*}{ Renda individual } & $\leq 1$ salário mínimo & 75,29 \\
\hline & > 1 salário mínimo & 24,71 \\
\hline \multirow{2}{*}{ Renda familiar } & $\leq 1$ salários mínimos & 16,5 \\
\hline & > 1 salários mínimos & 83,5 \\
\hline
\end{tabular}

No que diz respeito à avaliação cognitiva, todos os 85 idosos que participaram deste estudo apresentaram resultado abaixo da nota de corte, de acordo com os níveis de escolaridade no MEEM. Destes, 35,3\% apresentaram resultado de quatro a seis pontos abaixo da nota de corte.

Em relação à família dos idosos, a maioria dos domicílios é ocupada apenas por mais uma pessoa (35,3\%), em seguida são encontrados os idosos que moram com mais duas ou três pessoas $(21,2 \%$ e $10,6 \%$, respectivamente). Apenas $10,6 \%$ dos idosos residem sozinhos.

Além dos idosos, essas residências são ocupadas em sua maioria $(66,7 \%)$, pelos maridos ou esposas. Os resultados também mostram que como companheiros dos idosos estão seus filhos (13,3\%), irmãos $(6,7 \%)$, netos $(6,7 \%)$, mães $(3,3 \%)$ e cuidador $(3,3 \%)$.

A presença de domicílios multigeracionais foi verificada por meio da identificação de idosos que dividem seu domicílio com crianças de zero a 14 anos, representados por $21,2 \%$ dos idosos.
Nas diferentes regiões de vulnerabilidade social houve predomínio de residências que não apresentaram crianças como moradoras, no entanto, o número de idosos que compartilham suas casas com crianças é maior nas regiões de alta vulnerabilidade social (12,9\%), ou seja, em contextos de pobreza, quando comparado com as regiões de baixa vulnerabilidade $(8,2 \%)$.

Em contexto de alta e muito alta vulnerabilidade social observou-se predomínio de crianças do sexo feminino $(75,0 \%)$ e com faixa etária entre um e cinco anos (35,0\%). Apenas uma das crianças em idade escolar não frequentava a escola, e 25,0\% apresentavam algum problema de saúde.

Já em contexto de média, baixa e muito baixa vulnerabilidade social observou-se predomínio de crianças do sexo feminino $(55,5 \%)$, de faixa etária entre 11 e 14 anos (44,4\%). Todas as crianças em idade escolar frequentavam a escola e 33,3\% apresentavam algum problema de saúde.

Com relação ao apoio social avaliado pelo MOS, a média alcançada pelos 85 idosos, considerando as cinco dimensões de apoio social foi de 74,2 pontos com desvio 
padrão de 9,5 pontos. Analisando cada dimensão separadamente observa-se um bom nível de apoio social nas dimensões de apoio material e afetivo. Já as dimensões de apoio emocional, de informação e de interação social positiva demonstram um nível de apoio inferior, uma vez que estão abaixo da média total, como pode ser visualizado na Figura 1.
A análise de correlação entre os níveis de apoio social e a presença de crianças morando com idosos revelou uma correlação estatisticamente significativa entre a dimensão de apoio emocional e a presença de crianças no domicílio. A Tabela 2 apresenta a relação entre as cinco dimensões de apoio social e presença de crianças de zero a 14 anos no domicílio dos idosos.

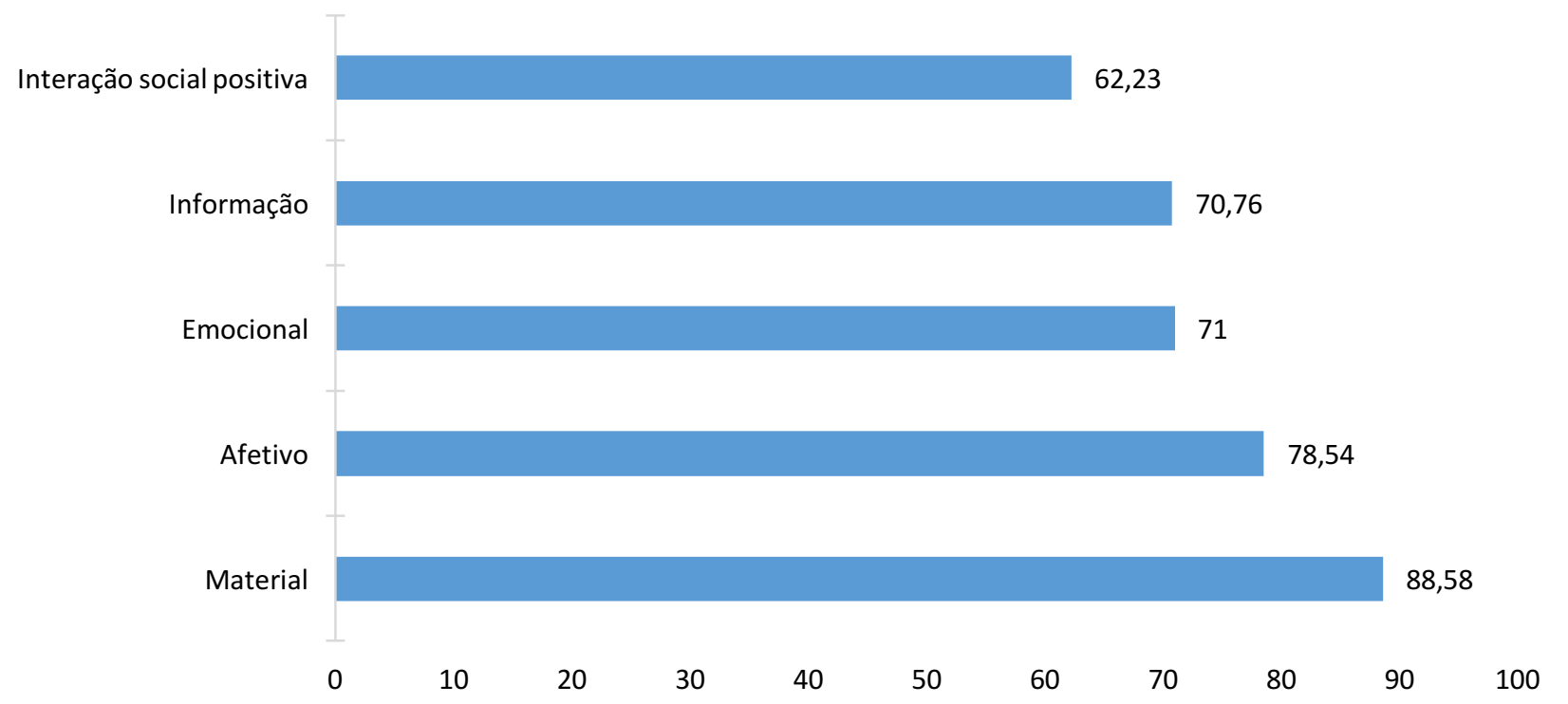

Figura 1: Escores médios alcançados pelos idosos em cada dimensão de apoio social. São Carlos, SP, Brasil, 2010.

Tabela 2: Relação entre as dimensões de apoio social e a presença de crianças no domicílio do idoso. São Carlos, SP, Brasil, 2010.

\begin{tabular}{|c|c|c|c|c|c|c|c|c|}
\hline & \multicolumn{3}{|c|}{ Presença } & \multicolumn{3}{|c|}{ Ausência } & \multicolumn{2}{|c|}{ Teste-t } \\
\hline & $\mathbf{N}$ & Média & D.P. & $\mathbf{N}$ & Média & D.P. & $\mathbf{T}$ & GI \\
\hline Apoio material & 18 & 92,22 & 16,55 & 67 & 87,61 & 19,23 & 1,01 & 30,52 \\
\hline Apoio afetivo & 18 & 80,36 & 21,62 & 67 & 78,05 & 21,89 & 0,4 & 27,12 \\
\hline Apoio emocional & 18 & 81,11 & 15,67 & 67 & 68,28 & 23,84 & $2,16^{*}$ & 83 \\
\hline Apoio de informação & 18 & 73,05 & 18,71 & 67 & 70,14 & 21,49 & 0,56 & 30,2 \\
\hline Apoio de interação social positiva & 18 & 69,16 & 21,43 & 67 & 60,37 & 23,5 & 1,51 & 21,97 \\
\hline
\end{tabular}

Nota: $* p<0,01$

\section{DISCUSSÃO}

No presente estudo a família dos idosos não se caracterizou como extensa, já que a maioria deles morava com somente mais uma pessoa na casa. Pouco mais de $10 \%$ dos idosos vivia sozinho, o que mostra que os idosos normalmente têm um ou mais companheiros(as). A porcentagem de crianças na casa dos idosos foi $21,2 \%$, sendo maior nas casas localizadas em área de alta e muito alta vulnerabilidade social (contextos de pobreza), podendo este agrupamento de gerações estar relacionado às necessidades tanto dos idosos, como das crianças, fato já relatado anteriormente ${ }^{(11)}$.

Buscando estudar o envelhecimento na percepção de 10 idosos frequentadores de um centro de convivência para a terceira idade, foi constatado que a figura dos netos e bisnetos é fonte de alegria e prazer para os idosos e alguns dizem preferir a convivência com jovens e crianças, por esta ser satisfatória ${ }^{(15)}$. Outro estudo que buscou analisar o significado da velhice para 48 idosos da zona rural do estado do Ceará constatou que os idosos 
relatam perdas e dependência, mas estão satisfeitos por poderem estar com filhos e netos ${ }^{(16)}$.

Dos 207 idosos que participavam de serviços relacionados à Universidade de Santa Cruz do Sul-SC, 85\% relataram que as relações familiares interferem no estado de humor/ânimo; e quando questionados sobre as relações que possuem com os netos, $79 \%$ relataram como muito boas, $19 \%$ como boas e $2 \%$ como regulares $^{(17)}$.

Em Taiwan, após avaliação de 4.582 idosos que tinham pelo menos um neto (menor de 18 anos de idade), verificou-se que $23,6 \%$ moravam com e cuidavam deste neto. Os avós cuidadores eram mais saudáveis e se disseram mais satisfeitos com o suporte emocional advindo da família e amigos do que os idosos não cuidadores $^{(18)}$. Os mesmos dados analisados longitudinalmente mostraram que essas associações podem variar dependendo do tempo de cuidado e do arranjo domiciliar dos avós, mas não são fatores causadores de melhores ou piores resultados, já que em todos os grupos, os cuidadores apresentaram resultados mais positivos do que os não cuidadores ${ }^{(19)}$.

No Chile, entre os 2.000 idosos entrevistados, $41 \%$ relataram residir com um ou mais netos e $50 \%$ ajudavam a cuidar do mesmo por quatro ou mais horas por semana. Os avôs que cuidavam dos netos por quatro ou mais horas por semana tiveram melhores níveis de satisfação com a vida dois anos depois; e os que forneciam apoio emocional, tiveram melhores escores no componente saúde mental em um questionário genérico de qualidade de vida no mesmo período. Para as avós, cuidar no neto por quatro ou mais horas semanais diminuiu o risco de depressão em dois anos ${ }^{(20)}$.

Uma investigação que buscou analisar a relação entre o contato com os netos na infância e esse neto ser parte da rede de relacionados dos avós na idade adulta identificou que uma maior frequência de contato, passar a noite juntos e cuidar do neto na infância foram fatores que contribuíram para que o neto fosse incluído como uma pessoa importante na rede de relacionamentos dos avós quando na idade adulta ${ }^{(21)}$. Assim, os benefícios do apoio social dos netos para com os avós na infância pode beneficiar o relacionamento entre os dois.

Residir com filhos e netos pode trazer benefícios para a relação do idoso com seus familiares e para a saúde do idoso. Por outro lado, morar com crianças não deve ser vista como garantia de velhice bem sucedida e nem de boas relações entre os familiares, uma vez que favorece trocas de apoio não balanceadas, pois pode haver uma tendência de que os filhos dispensem mais cuidado aos seus filhos do que aos seus pais ${ }^{(22)}$.

Assim como no presente estudo, os resultados do Estudo SABE apontam que o tipo de apoio social mais recebido pelos idosos foi o apoio material. No SABE, 92\% dos idosos do Brasil relatam receber apoio dos familiares, sendo mais citados os apoios de serviços, bens e financeiro. Em relação ao apoio fornecido pelos idosos, 88\% declaram fazê-lo, também citando serviços, bens e financeiro. Constatou-se que as condições de educação e financeiras aumentam a chance de oferecer ajuda e diminuem a de receber ${ }^{(23)}$.

Os idosos do presente estudo apresentaram maior nível de apoio emocional quando conviviam com uma criança na mesma casa, o que pode ser explicado pelas trocas afetivas relacionadas à avozidade. Quando o foco são os idosos e o bem estar familiar, a proximidade emocional aparenta ser o componente mais importante das relações intergeracionais ${ }^{(11)}$.

A relevância de se estudar o apoio social mediante o contato intergeracional está no fato de que apoio social satisfatório parece promover melhores condições de saúde, e que, a relação específica entre idosos e crianças proporciona uma troca de experiências entre as gerações, já citada em trabalhos anteriores ${ }^{(15,24)}$. Além disso, a necessidade de ajuda material, afetiva e física pode levar os idosos a morarem com suas famílias, inclusive os netos $^{(4)}$, sendo que o maior apoio emocional percebido pelos idosos que moravam com crianças no presente estudo pode ajudar o idoso a lidar com as atividades do dia a dia. 


\section{CONCLUSÃO}

Dos 85 idosos avaliados, a maioria era mulher, entre 70-79 anos, analfabeta e viúva. Todos os idosos possuíam alteração cognitiva, sendo que a maior parte apresentou resultado de quatro a seis pontos abaixo da nota de corte no MEEM. Na maioria dos casos, os idosos residiam com mais uma pessoa na casa, sendo marido ou esposa. Os domicílios multigeracionais foram verificados pela presença de crianças na casa, o que ocorreu em $21,2 \%$ dos casos, sendo que os idosos que moram com crianças apresentam melhor nível de apoio emocional do que os que não moram. Os resultados revelaram bons níveis de apoio social recebidos pelos idosos e correlação estatisticamente significativa entre a dimensão de apoio emocional e a presença de crianças no domicílio $(t=2,16$, sendo $p<0,01)$.

Considerando-se que estudos nessa temática são escassos na literatura, os resultados obtidos contribuirão com o cuidado aos idosos, uma vez que o contato entre estas diferentes gerações demonstrou ser benéfico, e que ao conhecer a relação do idoso com sua família, especificamente com as crianças que convivem com ele, vislumbra-se a possibilidade de utilizar a relação intergeracional a favor do planejamento e desenvolvimento de linhas de cuidado à população idosa.

A realização de novos estudos deve ser encorajada com uma amostra mais representativa, e que utilizem delineamentos de pesquisa que proporcionem a comparação entre grupos de idosos que moram e que não moram com criança; e que possuam ou não alterações cognitivas, a fim de aprofundar o conhecimento a respeito da relação entre idosos e crianças.

\section{Agradecimentos e Financiamento}

Agradecemos ao Programa de Pós-Graduação em Enfermagem da UFSCar e ao financiamento da Coordenação de Aperfeiçoamento de Pessoal de Nível Superior (CAPES) pela possibilidade de desenvolvimento desse estudo.

8. Atkins J, Naismith SL, Luscombe GM, Hickie IB. Psychological distress and quality of life in older persons: relative contributions of fixed and modifiable risk factors. BMC Psychiatry. 2013;13(1):249.

9. D'orsi E, Xavier AJ, Ramos LR. Trabalho, suporte social e lazer protegem idosos da perda funcional: estudo Epidoso. Rev. Saúde Pública; 2011;45(4):85-92.

10. Machado JC, Ribeiro RCL, Leal PFG, Cotta RMM. Avaliação do declínio cognitivo e sua relação com as características socioeconômicas dos idosos em Viçosa-MG. Rev Bras Epidemiol. 2007;10(4):592-605.

11. Rabelo DF, Neri AL. A complexidade dos relacionamentos intergeracionais e a saúde mental dos idosos. Pensando Famílias. 2014;18(1):138-53.

12. Andrade CR. Associação entre apoio social e frequência relatada de auto-exame das mamas no Estudo Pró-Saúde [dissertação]. Rio de Janeiro: Escola Nacional de Saúde Pública/Fundação Oswaldo Cruz; 2001. 66p.

13. Folstein MF, Folstein SE, McHugh PR. "Mini-Mental State": a Practical Method for Grading the Cognitive State of Patients for the Clinician. J Psychiatr Res. 1975;12:189-98.

14. Nitrini R, Caramelli P, Bottino CMC, Damasceno BP, Brucki $\mathrm{SMD}$, Anginah R. Diagnóstico de doença de Alzheimer no Brasil: avaliação cognitiva e funcional. Recomendações do Departamento Científico de Neurologia Cognitiva e do 
Envelhecimento da Academia Brasileira de Neurologia. Arq Neuropsiquiatr. 2005;63(3A): 720-7.

15. Silva EV, Martins F, Bachion MM, Nakatani AYK.. Percepção de idosos de um centro de convivência sobre envelhecimento. Rev Min Enfermagem. 2006;10(1):46-53.

16. Freitas MC, Queiroz TA, Sousa JAV. O significado da velhice e da experiência de envelhecer para os idosos. Rev Esc Enferm USP. 2010;44(2):407-12.

17. Areosa SVC, Benitez LB, Wichmann FMA. Relações familiares e o convívio social entre idosos. Textos \& Contextos (Porto Alegre). 2012;11(1):184-192.

18. Ku LE, Stearns SC, Van Houtven CH, Holmes GM. The health effects of caregiving by grandparents in Taiwan: an instrumental variable estimation. Rev Econ Household. 2012;10:521-40.

19. Ku LE, Stearns SC, Van Houtven CH, Lee SD, DilworthAnderson $\mathrm{P}$, Konrad TR. Impact of caring for grandchildren on the health of grandparents in Taiwan. J Gerontol B Psychol Sci Soc Sci. 2013;68(6):1009-21.

20. Grundy EM, Albala C, Allen E, Dangour AD, Elbourne D, Uauy R. Gradparenting and psychosocial health among older Chileans: a longitudinal analysis. Aging Ment Health. 2012;16(6):1047-57.

21. Geurts T, Tilburg TGV, Poortman AR. The grandparentgrandchild relationship in childhood and adulthood: a matter of continuation? Personal Relationships. 2012;19(2):267-278. 22. Fingerman KL, Sechrist J, Birditt K. Changing views on intergenerational ties. Gerontology. 2013;59(1):64-70. 23. Saad PM. Transferencias informales de apoyo de los adultos mayores en América Latina y el Caribe: estudio comparativo de encuestas SABE. Notas Poblacion. 2003;3(77):175-217.

24. Leder S, Grinstead LN, Torres E. Grandparents Raising Grandchildren: Stressors, Social Support, and Health Outcomes. J Fam Nurs. 2007;13(3):333-52.

Recebido: 26/07/2013.

Aceito: $18 / 03 / 2015$.

Publicado: 30/09/2015. 